\title{
EL BONO SOCIAL Y LAS COOPERATIVAS ENERGÉTICAS VERDES: SITUACIÓN Y PERSPECTIVAS ${ }^{1}$
}

\author{
POR \\ Egoitz JAIO GABIOLA, \\ Juan Diego PAREDES GÁZQUEZ y \\ José Antonio SÁNCHEZ RODRÍGUEZ ${ }^{2}$
}

\section{RESUMEN}

Las tarifas sociales son, junto con la transferencia de rentas y las medidas de eficiencia energética, uno de los instrumentos para paliar la pobreza energética. A la tarifa social española, creada en el año 2009 se le denomina "bono social". Para acceder al bono social, aparte de cumplir los requisitos socioeconómicos estipulados por ley, hay que tener contratada la electricidad con alguna comercializadora de referencia, las cuales tienen la obligación de ofrecer el bono social. Las cooperativas energéticas verdes, un fenómeno reciente, al no ser comercializadoras de referencia, no están obligadas a ofrecer el bono social. Esto no quiere decir que entre los socios de estas cooperativas no haya personas vulnerables o en riesgo de pobreza energética. Este trabajo centra su análisis en dos objetivos. El primero es trazar el perfil socioeconómico del socio de las cooperativas verdes. El segundo es observar si estos socios tienen derecho al bono social actual, así como a otras tarifas sociales alternativas con requisitos diferentes a los del bono social. Para ello, se realiza una encuesta a los socios de la mayor cooperativa energética verde de España. Los resultados evidencian que sí existe riesgo de pobreza energética entre los socios de las cooperativas energéticas verdes, si bien su incidencia depende de cómo se defina al consumidor vulnerable.

Palabras clave: Sociedades cooperativas, tarifas sociales, pobreza energética, electricidad, energías renovables

\footnotetext{
${ }^{1}$ Los autores desean agradecer la colaboración de los socios de Som Energia por participar en la encuesta, así como los consejos y aportaciones de D. Santiago Ochoa de Eribe, Director de GoiEner.

${ }_{2}^{2}$ Facultad de Ciencias Económicas y Empresariales, Universidad Nacional de Educación a Distancia. Direcciones de correo electrónico: juandiegoparedes@cee.uned.es, ejaio@ cee.uned.es y jasanchez@ cee.uned.es. REVESCO No 122 - Tercer Cuatrimestre 2016 - ISSN: 1885-8031 - www.ucm.es/info/revesco

http://dx.doi.org/10.5209/rev_REVE.2016.v122.52024

Fecha de recepción: 06/11/2015

Fecha de aceptación: 01/03/2016
} 
Claves ECONLIT: I31, P13, Q01, Q42.

\title{
THE SPANISH SOCIAL TARIFF AND RENEWABLE ENERGY COOPERATIVES: SITUATION AND PERSPECTIVES
}

\begin{abstract}
Social tariffs are, along with transfer payments and energy efficiency measures, an instrument to alleviate energy poverty. The name of the Spanish social tariff is "bono social", and it was established in 2009. To qualify for bono social, the electricity consumer should meet any of the socioeconomic requirements stipulated by law and contract the electricity supply with a "comercializadora de referencia", companies that are required to offer the bono social by law. Renewable energy cooperatives, a recent phenomenon in Spain, are not comercializadoras de referencia, so they are not obliged to offer the bono social. This does not mean there are no cooperative members at risk of energy poverty or vulnerable consumers. This study has two objectives. The first is to sketch the socioeconomic profile of members of the renewable energy cooperatives. The second is to analyze if these members are entitled to the bono social, or would be to other subsidized prices with different requirements to those of the bono social. For this purpose, we conducted a survey to members of the largest renewable energy cooperative in Spain, Som Energia. The results show that the members of renewable energy cooperatives are exposed to energy poverty risk, although its reach depends on the definition of vulnerable consumer.
\end{abstract}

Keywords: Cooperative societies, social tariffs, energy poverty, electricity, renewable energies

\section{INTRODUCCIÓN}

Las tarifas sociales eléctricas pretenden mitigar el esfuerzo que deben hacer aquellos consumidores considerados vulnerables al pagar la factura eléctrica. En España esa tarifa social se llama "bono social", y fue aprobada a través del Real Decreto-ley 6/2009, de 30 de abril. Sin embargo, a pesar de la crisis económica y de que el precio de la electricidad ha aumentado considerablemente en los últimos años, los beneficiarios del bono social han disminuido año tras año.

En este contexto, las socias y socios consumidores (en adelante socios) de las cooperativas energéticas verdes o cooperativas de consumo de energía renovable se 
encuentran en una situación particular. Por un lado, la legislación no obliga a las cooperativas energéticas verdes a ofertar el bono social a sus socios. Por otro lado, se desconoce con exactitud cuál es el perfil socioeconómico de los socios de estas cooperativas y si efectivamente tendrían derecho a solicitar el bono social.

Este trabajo tiene por objetivo trazar el perfil socioeconómico de los socios de las cooperativas energéticas verdes, así como analizar si son consumidores vulnerables o no. En particular, se estudia el caso Som Energia, la mayor cooperativa energética verde de España, en cuatro escenarios en los que los requisitos socioeconómicos para acceder a una tarifa social son diferentes.

El trabajo se estructura en cinco apartados principales. Tras esta introducción, el segundo apartado describe la tarifa social española: el bono social. El tercer apartado describe el estado de las cooperativas energéticas en España, prestando especial atención al acceso a tarifas sociales en las cooperativas energéticas verdes y planteando cuatro escenarios de tarifas sociales. En el siguiente apartado se detalla el diseño de la investigación, y en el quinto apartado se exponen y discuten los resultados. Por último, las conclusiones del trabajo se recogen en el sexto apartado.

\section{LA POBREZA ENERGÉTICA Y LA TARIFA SOCIAL ESPAÑOLA: EL BONO SOCIAL}

El origen de las tarifas sociales está estrechamente relacionado con la pobreza energética, entendida esta, según el Dictamen de Iniciativa 2013/C 341/05 de 21 de noviembre de 2013, como la dificultad o la incapacidad de mantener la vivienda en unas condiciones adecuadas de temperatura, así como de disponer de otros servicios energéticos esenciales a un precio razonable. De acuerdo con Tirado Herrero et al. (2014), la pobreza energética ha aumentado en España en los últimos años: el porcentaje de hogares que se declaraban incapaces de mantener su vivienda a una temperatura adecuada en invierno aumento un 19\% en el periodo 2010-2012. Entre las causas de este incremento se identifican:

- El aumento del desempleo y el descenso de los salarios.

- La baja eficiencia energética del parque de viviendas.

- El aumento del precio de la electricidad. La proporción de gastos destinados a pagar el suministro de energía ha pasado de un 4,3\% en 2007 al 6,5\% en 2012. 
A estas tres causas principales hay que añadir la baja educación en cuanto al consumo energético. Actualmente, el mayor potencial de ahorro energético se encuentra en concienciar a los consumidores y en formarles sobre cómo podrían llevar a cabo ese ahorro (Moreno et al., 2014).

La Directiva 2009/72/CE de 13 de julio de 2009 dictamina que los Estados miembros deben garantizar el suministro de energía necesaria a consumidores vulnerables o en riesgo de pobreza energética, quedando la definición de este tipo de clientes en manos de los Estados miembros. Las tarifas sociales son, junto con la transferencia de rentas o las medidas de eficiencia energética, instrumentos para garantizar este suministro de energía. Los requisitos para acceder a la tarifa social varían de un país a otro y pueden cambiar a lo largo del tiempo.

La tarifa social eléctrica española es el bono social. Este bono, incluyendo su financiación, se aprobó en plena crisis económica a través del Real Decreto-ley 6/2009, de 30 de abril.

\subsection{Funcionamiento y estado del bono social}

La financiación del bono social la comparten las empresas titulares de instalaciones de generación del sistema eléctrico. Para acceder al bono social, el art. 45.2 del Real Decreto-ley 6/2009, de 30 de abril, establece que ha de cumplirse al menos uno de los siguientes requisitos socioeconómicos:

- Tener una potencia contratada inferior a 3 kilovatios $(\mathrm{kW})$ en su vivienda habitual.

- Tener 60 o más años de edad y ser pensionista del Sistema de la Seguridad Social por jubilación, incapacidad permanente y viudedad, percibiendo por ello la cuantía mínima vigente en cada momento para dichas clases de pensión con respecto a los titulares con cónyuge a cargo o a los titulares sin cónyuge que viven en una unidad económica unipersonal.

- Tener 60 o más años de edad y ser beneficiario de pensiones del extinguido Seguro Obligatorio de Vejez e Invalidez y de pensiones no contributivas de jubilación e invalidez.

- Ser familia numerosa.

- Formar parte de una unidad familiar que tenga a todos sus miembros en situación de desempleo. 
Los miembros de estos colectivos son los llamados consumidores vulnerables. Así mismo, para entender el bono social, es menester introducir el concepto de la Tarifa de Último Recurso (TUR). La TUR estuvo vigente desde el 1 de enero del 2009 hasta el 31 de mayo de 2014. Era el precio máximo que las comercializadoras de último recurso (CUR) podían cobrar a sus clientes. La TUR estaba regulada por el Ministerio de Industria, Turismo y Comercio, y tenían acceso a ella todos los consumidores conectados a baja tensión con una potencia contratada igual o inferior a $10 \mathrm{~kW}$. Estos consumidores podían acogerse al mercado regulado y acceder a la TUR, o aceptar alguna otra oferta y pasarse al mercado liberalizado, renunciando así a su derecho a la TUR.

La TUR solo la ofrecían las CUR. A las personas que tenían derecho al bono social se les congeló la tarifa eléctrica, esto es, el bono social cubría la diferencia entre el valor de la TUR y un valor de referencia, que se denominaba tarifa reducida. Este valor de referencia era el precio de la electricidad a 1 de julio de 2009. Por tanto, conforme aumentaba el coste de la electricidad, aumentaba la cuantía que las empresas titulares de instalaciones de generación del sistema eléctrico tenían que destinar a financiar el bono social.

Con la entrada en vigor del Real Decreto 216/2014, de 28 de marzo, las CUR pasan a llamarse comercializadoras de referencia y la TUR se renombra como Precio Voluntario al Pequeño Consumidor. Además, el bono social pasa a ser un descuento de un $25 \%$ en todos los términos de la factura. Con los aumentos de las tarifas de luz que ha habido durante estos años, este cambio implica una disminución en la cuantía que se destina a financiar el bono social.

Otro aspecto que ha estado a punto de sufrir modificaciones han sido los requisitos que se deben cumplir para acceder al bono social. Aparte de cumplir alguno de los requisitos actuales para acceder al bono social, el Proyecto de Real Decreto por el que se regula la actividad de comercialización y las condiciones de contratación y suministro de energía eléctrica de 19 de julio de 2013, establecería los siguientes límites salariales para la unidad familiar: para una persona el 120\% del Salario Mínimo Interprofesional (10.841€), para la segunda persona el 70\% (7.588€) y para la tercera y siguientes el 50\% (5.420€). Con esta reforma parte de los beneficiarios actuales se quedarían sin poder acceder al bono social. Dicha modificación, que se planteó en la reforma eléctrica de julio de 2013, sigue pendiente de aprobación. 
En la tabla 1 se observa la evolución de los beneficiarios del bono social desde su implantación en el 2009 hasta el año 2014. El número de beneficiarios ha descendido un 17,88\% en total desde que comenzó a aplicarse. La disminución se debe a que los contratos con una potencia inferior a $3 \mathrm{~kW}$ han disminuido considerablemente, aunque los miembros de este colectivo alcanzan casi el $80 \%$ del total de beneficiarios.

Tabla 1. Evolución del bono social 2009-2014

\begin{tabular}{|c|c|c|c|c|c|}
\hline Año & $\begin{array}{c}\text { Potencia } \\
<3 k W\end{array}$ & Desempleados & $\begin{array}{c}\text { Familia } \\
\text { numerosa }\end{array}$ & $\begin{array}{c}\text { Pensiones } \\
\text { mínimas }\end{array}$ & Total \\
\hline $\mathbf{2 0 0 9}$ & 2.646 .928 & 15.032 & 64.832 & 277.815 & 3.004 .607 \\
\hline $\mathbf{2 0 1 0}$ & 2.402 .511 & 23.7766 & 100.581 & 317.205 & 2.844 .073 \\
\hline $\mathbf{2 0 1 1}$ & 2.169 .869 & 31.067 & 118.611 & 307.893 & 2.627 .440 \\
\hline $\mathbf{2 0 1 2}$ & 2.067 .585 & 33.735 & 129.466 & 283.790 & 2.513 .896 \\
\hline $\mathbf{2 0 1 3}$ & 1.986 .822 & 48.881 & 150.872 & 283.790 & 2.470 .365 \\
\hline $\mathbf{2 0 1 4}$ & 1.961 .179 & 54.824 & 162.174 & 289.113 & 2.467 .290 \\
\hline $\mathbf{2 0 1 4 / 2 0 0 9}$ & $\mathbf{- 2 5 , 9 1 \%}$ & $\mathbf{2 6 4 , 7 2 \%}$ & $\mathbf{1 5 0 , 1 4 \%}$ & $\mathbf{4 , 0 7 \%}$ & $\mathbf{- 1 7 , 8 8 \%}$ \\
\hline
\end{tabular}

Fuente: CNMC (2014)

\subsection{Críticas al bono social}

Las críticas al bono social se han centrado principalmente en dos puntos: los requisitos de acceso y el mecanismo de financiación. Respecto a los requisitos de acceso, pueden discutirse los siguientes aspectos:

- Tener una potencia contratada inferior a $3 \mathrm{~kW}$ en su vivienda habitual. Puede haber familias que con $3 \mathrm{~kW}$ no puedan cubrir sus necesidades energéticas básicas.

- Pensionistas con 60 o más años que perciban la pensión mínima por jubilación permanente, incapacidad o viudedad. Este colectivo puede obtener ingresos adicionales a través de otros activos.

- Ser familia numerosa. Una familia numerosa puede tener unos ingresos económicos altos, todo depende de la renta media de los miembros de la familia.

Los requisitos para acceder al bono social no tienen en cuenta criterios económicos como pueden ser los ingresos familiares. En este sentido, al contrario de lo que sostiene Marín Uribe (2009), el bono social no está protegiendo a los consumidores más vulnerables de las subidas de la tarifa eléctrica. Puesto que la pérdida de poder adquisitivo de las familias es una de las principales causas del aumento de la pobreza energética (Tirado Herrero et al., 2014), redefinir el concepto de consumidor vulnerable es una prioridad. 
Respecto a la financiación del bono social, si bien en principio esta responsabilidad recae sobre las empresas titulares de instalaciones de generación del sistema eléctrico, en última instancia son todos los consumidores de electricidad los que, a través de la factura eléctrica, financian el bono social. Desde el centro de investigación Economics for Energy apuestan por trasladar dicha financiación a los presupuestos públicos (Romero et al., 2014). Así mismo, Romero et al. (2014) señalan la posibilidad de canalizar el bono social como una transferencia de tanto alzado y no como un descuento, modulada en función de las características del hogar y utilizable únicamente para pagar los gastos energéticos.

\section{LAS COOPERATIVAS ENERGÉTICAS Y LAS COOPERATIVAS ENERGÉTICAS VERDES}

Las cooperativas energéticas nacieron en Europa en las primeras décadas del siglo XX, principalmente en Dinamarca, Alemania y Reino Unido. El objetivo principal de estas cooperativas era abastecer a los pueblos apartados de los núcleos urbanos adonde era difícil hacer llegar el suministro eléctrico (Arnáez Arce, 2011).

En España existían antes de la Guerra Civil más de 2.000 cooperativas energéticas cuyo propósito era hacer llegar el suministro eléctrico a zonas rurales (Pérez Lucía, 2015). Actualmente existen una veintena de ellas, principalmente en la Comunidad Valenciana, aunque también hay en las comunidades de Andalucía, Aragón, Cataluña, Madrid y el País Vasco (tabla 2). La práctica totalidad de ellas son distribuidoras y comercializadoras. A día de hoy, de las 336 distribuidoras de electricidad existentes en España, 21 son sociedades cooperativas. Así mismo, de las 302 comercializadoras de electricidad existentes España, 21 son cooperativas.

Tabla 2. Cooperativas energéticas en España

\begin{tabular}{|l|c|c|}
\hline \multicolumn{1}{|c|}{ Cooperativa energética } & Lugar & $\begin{array}{c}\text { Año de } \\
\text { creación }\end{array}$ \\
\hline Cooperativa Eléctrica Benéfica Albaterense S.C.V & Albatera (Alicante) & 1929 \\
\hline Cooperativa Eléctrica Benéfica Catralense S.C.V & Catral (Alicante) & 1927 \\
\hline Cooperativa Eléctrica de Castellar S.C.V & Valencia & 1924 \\
\hline $\begin{array}{l}\text { Cooperativa Popular de Fluido Eléctrico de } \\
\text { Camprodon S.C.C.L.* }\end{array}$ & Camprodon (Girona) & 1944 \\
\hline $\begin{array}{l}\text { Cooperativa Valenciana Electro Distribuidora de } \\
\text { Fuerza y Alumbrado Serrallo S.C.V }\end{array}$ & $\begin{array}{c}\text { Grao de Castellón } \\
\text { (Castellón) }\end{array}$ & 1965 \\
\hline Eléctrica Algimia de Alfara S.C.V & $\begin{array}{c}\text { Algimia de Alfara } \\
\text { (Valencia) }\end{array}$ & - \\
\hline
\end{tabular}

REVESCO No 122 - Tercer Cuatrimestre 2016 - ISSN: 1885-8031 - www.ucm.es/info/revesco 


\begin{tabular}{|l|c|c|}
\hline Eléctrica de Callosa de Segura S.V.L. & $\begin{array}{c}\text { Callosa de Segura } \\
\text { (Alicante) }\end{array}$ & 1929 \\
\hline Eléctrica de Chera S.C.V & Chera (Valencia) & 1928 \\
\hline Eléctrica de Guadassuar S.C.V & Guadassuar (Valencia) & 1931 \\
\hline Eléctrica de Meliana S.C.V & Meliana (Valencia) & 1922 \\
\hline Eléctrica de Pozo S.C.M. & Madrid (Madrid) & - \\
\hline Eléctrica de Sot de Chera S.C.V & $\begin{array}{c}\text { Sot de Chera } \\
\text { (Valencia) }\end{array}$ & 1943 \\
\hline Eléctrica de Vinalesa S.C.V. & Vinalesa (Valencia) & 1911 \\
\hline Eléctrica Ntra. Sra. De Gracia S.C.V & Biar (Alicante) & 1924 \\
\hline Eléctrica Popular S.C.M. & Perales de Tajuna & - \\
\hline $\begin{array}{l}\text { Electrodistribuidora de Fuerza y Alub. } \\
\text { “Casablanca” S.C.V }\end{array}$ & Almenara (Castellón) & 1950 \\
\hline Electroharinera Belsetana S.C.* & Bielsa (Huesca) & - \\
\hline Fluido Eléctrico de Museros S.C.V & Museros (Valencia) & 1913 \\
\hline GoiEner S.C. ** & Ordizia (Gipuzkoa) & 2010 \\
\hline $\begin{array}{l}\text { Grupo de Electrización Rural de Binéfar y } \\
\text { Comarca S.C. * }\end{array}$ & Binéfar (Huesca) & - \\
\hline $\begin{array}{l}\text { Benéfica de Cons. de Elect. “San Francisco de } \\
\text { Asis” de Creu S.C.V }\end{array}$ & Crevillent (Alicante) & 1925 \\
\hline Som Energia S.C.C.L.** & Girona & 2010 \\
\hline Suministros especiales Alginetenses S.C.V & Alginet (Valencia) & 1930 \\
\hline Zencer S.C.A. ** & Fuengirola (Málaga) & 2012 \\
\hline
\end{tabular}

*Cooperativas que son sólo distribuidoras

** Cooperativas que son sólo comercializadoras

Fuente: elaboración propia a partir de CNMC (2015), MINET (2015) y páginas web de cada cooperativa

En los últimos años han aparecido cooperativas energéticas verdes (CEV) o cooperativas de consumo de energía renovable (tabla 3). Estas cooperativas sin ánimo de lucro comercializan una energía $100 \%$ renovable, la denominada electricidad verde, que es correspondientemente certificada. Esta electricidad se comercializa a partir del sistema de utilización de los certificados de garantía de origen. La Comisión Nacional de la Energía es quien tiene la potestad de expedir certificados que ratifican que la electricidad generada por una central es de origen renovable. Las CEV pueden beneficiarse de la tendencia hacia modelos de consumo más respetuosos con el medioambiente, por lo que se espera que tanto el número de $\mathrm{CEV}$ como el de sus socios crezcan en los próximos años. 
Tabla 3. Cooperativas energéticas verdes en España

\begin{tabular}{|l|c|c|c|c|}
\hline $\begin{array}{c}\text { Cooperativa energética } \\
\text { verde }\end{array}$ & Lugar & $\begin{array}{c}\text { Año de } \\
\text { creación }\end{array}$ & Socios & Contratos \\
\hline GoiEner S.C. & Ordizia (Gipuzkoa) & 2010 & 3590 & 3598 \\
\hline Megara S.C.C.L.* & Soria & 2015 & - & - \\
\hline Nosa Energia S.C.G.* & $\begin{array}{c}\text { Vilagarcia de Arousa } \\
\text { (Pontevedra) }\end{array}$ & 2015 & 138 & 59 \\
\hline Solabria S.C.* & Torrelavega (Santander) & 2015 & - & - \\
\hline Som Energia S.C.C.L. & Girona & 2010 & 22045 & 28206 \\
\hline Zencer S.C.A. & Fuengirola (Málaga) & 2012 & - & - \\
\hline
\end{tabular}

* Cooperativas energéticas verdes que aún no están registradas como comercializadoras en al CNMC Fuente: Elaboración propia

Las CEV surgen con un propósito diferente al de aquellas cooperativas energéticas que vieron luz a principios del siglo pasado. Si en aquel entonces, el objetivo fundamental era hacer llegar el suministro eléctrico a zonas rurales donde a las empresas eléctricas no les resultaba rentable, ahora el objetivo es contribuir al cambio de modelo energético. Por ejemplo, en la web de Som Energia, la mayor CEV de España, afirman que:

"Estamos comprometidos a impulsar un cambio del modelo energético actual para conseguir un modelo $100 \%$ renovable. Porque el modelo energético actual basado en combustibles fósiles es insostenible" 3

El objetivo de GoiEner, CEV vasca con más de 3.600 socios, apunta en la misma dirección que el de Som Energia, tal y como leemos en su página web:

"GoiEner es un proyecto cooperativo de generación y consumo de energía renovable con el que se quiere recuperar la soberanía energética" 4

"El objetivo de GoiEner es convertirse en una cooperativa sin ánimo de lucro que reúna a miles de personas con el deseo de cambiar el modelo energético actual y trabajar para alcanzar un modelo $100 \%$ renovable" 5

\subsection{Tarifas sociales en las cooperativas verdes}

Las CEV, al no ser comercializadoras de referencia, no están obligadas a ofrecer el bono social. No obstante, pueden ofrecerlo voluntariamente, ya sea uno con las mismas

\footnotetext{
${ }^{3}$ https://www.somenergia.coop/es/quienes-somos/

4 http://www.goiener.com/la-cooperativa/

5 http://www.goiener.com/faq/
} 
características de bono social vigente u otra tarifa social. A día de hoy podemos afirmar que ninguna CEV ofrece alguna cualquier tarifa social a sus socios vulnerables, lo que podría deberse hasta a tres razones:

- Dada su reciente creación, aún no se lo han planteado.

- Los beneficios económicos todavía son escasos.

- No es una prioridad para las cooperativas.

Si se quiere cambiar el modelo energético, apostar por las energías renovables y concienciar que se puede reducir el consumo energético actual, hay que pensar en toda la población, incluyendo a aquellos que actualmente tienen problemas para cubrir sus necesidades energéticas. Estos problemas pueden darse no sólo por los motivos socioeconómicos cubiertos por el bono social, sino también por otros para los que actualmente no tiene cobertura.

Las personas en riesgo de pobreza energéticas pueden tener varias dificultades a la hora de asociarse a una CEV. Podría darse el caso de consumidores que en su día optaron por estas cooperativas sin tener problemas para cubrir sus necesidades energéticas, pero que actualmente necesitan una tarifa social. Además, para hacerse socio de estas cooperativas hay que realizar una aportación de $100 €$ que puede actuar como una barrera de entrada para aquellas personas que, aun compartiendo la filosofía de las CEV, no puedan permitírselo. No obstante, es posible encontrar casos de consumidores que, estando en una situación de vulnerabilidad, aun quieran contribuir a cambiar el modelo energético y se asocien a estas cooperativas.

Por tanto, resulta de interés estudiar cuál es el perfil socioeconómico de los socios de las CEV, y si están en condiciones de solicitar una tarifa social aun cuando la CEV no tenga la obligación de proporcionársela. Respecto a este último punto, se plantean hasta cuatro escenarios con tarifas sociales diferentes: dos de ellos están basados en situaciones reales de tarifas sociales, otros en un proyecto de ley y otro exclusivamente en requisitos económicos. Cada escenario exige unos requisitos socioeconómicos y económicos diferentes a los socios de las CEV. De este modo se pretende observar qué porcentaje de socios sería vulnerable en cada escenario, así como los cambios que se producen en tal cifra al alterarse los requisitos de acceso a la tarifa social. Los cuatro posibles escenarios que se proponen se detallan a continuación. 


\section{Escenario 1. Bono social actual}

En este escenario los requisitos de acceso al bono social son los fijados en el Real Decreto-ley 6/2009, de 30 de abril.

\section{Escenario 2. Proyecto de bono social con requisitos mixtos}

En este escenario se da importancia a los requisitos económicos para acceder al bono social, si bien también se consideran los requisitos que exige el bono social actual. Para acceder al bono social habrían de cumplirse dos condiciones:

1. Alguno de los cuatro requisitos fijados en el Real Decreto-ley 6/2009, de 30 de abril.

2. Un requisito adicional de umbral de renta, conforme a lo establecido en el Proyecto de Real Decreto por el que se regula la actividad de comercialización y las condiciones de contratación y suministro de energía eléctrica de 19 de julio de 2013. Estos umbrales de ingresos para la unidad familiar serían: para una persona el 120\% del Salario Mínimo Interprofesional (10.841€), para la segunda persona el 70\% (7.588€) y para la tercera y siguientes el 50\% (5.420€).

\section{Escenario 3. Bono social con requisitos económicos}

En este escenario el acceso al bono social depende exclusivamente de criterios económicos, en concreto de los umbrales de renta fijados en el Proyecto de Real Decreto por el que se regula la actividad de comercialización y las condiciones de contratación y suministro de energía eléctrica de 19 de julio de 2013.

\section{Escenario 4. Tarifa social alternativa}

En este escenario se consideran tarifas sociales que ya están siendo aplicadas en el sector eléctrico. Uno de los casos más reseñables es Electra Caldense Energia S.A. La empresa Electra Caldense Energia S.A., a pesar de no ser una comercializadora de referencia, y por tanto no tener la obligación de ofrecer el bono social, ha venido ofreciéndolo desde sus inicios. En un principio, siempre y cuando los consumidores cumplían los requisitos de acceso al bono social, la compañía les factura como si estuvieran en una comercializadora de referencia. Más tarde, la empresa, los servicios sociales municipales y el ayuntamiento de Caldes de Montbui llegaron a un acuerdo para modificar los requisitos de acceso a la bonificación y firmaron un convenio para crear una tarifa solcial basada en la renta neta 
disponible de los consumidores. La tarifa social alternativa tiene los siguientes requisitos de acceso para los consumidores:

- Tener un contrato de suministro con Electra Caldense Energia S.A

- Estar empadronado en Caldes de Montbui.

- Que la renta mensual neta por miembro de la unidad familiar no supere los 227,64€.

Esta tarifa social alternativa se ofrece desde el 1 de enero del 2015. Para el cálculo de la renta mensual neta, los consumidores se pueden deducir hasta un máximo de $600 €$ mensuales en concepto de alquiler o crédito hipotecario, estableciéndose además un límite máximo de consumo de $5.500 \mathrm{kWh} /$ año. Al añadir el gasto hipotecario o del alquiler se hace más real el cálculo de una posible vulnerabilidad económica. Excepcionalmente, si los servicios sociales lo consideran oportuno, pueden acceder a la tarifa social alternativa consumidores que no cumplan estos requisitos.

\section{Comparación de escenarios}

La tabla 4 compara los requisitos de acceso a las tarifas sociales en cada uno de los escenarios planteados.

Tabla 4. Comparativa de escenarios de tarifa social

\begin{tabular}{|l|c|c|c|c|}
\hline \multicolumn{1}{|c|}{ Requisito } & $\begin{array}{c}\text { 1. Bono social } \\
\text { actual }\end{array}$ & $\begin{array}{c}\text { 2. Proyecto de } \\
\text { bono social } \\
\text { con requisitos } \\
\text { mixtos }\end{array}$ & $\begin{array}{c}\text { 3. Bono social } \\
\text { con requisitos } \\
\text { económicos }\end{array}$ & $\begin{array}{c}\text { 4. Tarifa } \\
\text { social } \\
\text { alternativa }\end{array}$ \\
\hline $\begin{array}{l}\text { Potencia inferior a } \\
3 \mathrm{~kW}\end{array}$ & $\checkmark$ & $\checkmark$ & & \\
\hline Desempleados & $\checkmark$ & $\checkmark$ & & \\
\hline Familia numerosa & $\checkmark$ & $\checkmark$ & & \\
\hline $\begin{array}{l}\text { Pensiones } \\
\text { mínimas }\end{array}$ & $\checkmark$ & $\checkmark$ & & $\checkmark^{*}$ \\
\hline Umbral de renta & & $\checkmark$ & $\checkmark$ & \\
\hline
\end{tabular}

* Requisito de umbral de renta al que se pueden deducir costes de alquiler o crédito hipotecario. Fuente: elaboración propia

\section{DISEÑO DE LA INVESTIGACIÓN}

El objetivo de esta investigación es doble. En primer lugar, se pretende observar las características de los socios de las CEV. En segundo lugar, se analizan y comparan los cuatro escenarios de tarifa social planteados: bono social actual, proyecto de bono social con 
requisitos mixtos, bono social con requisitos económicos y tarifa social alternativa. De este modo, esta investigación permitirá estimar qué porcentaje de socios de las CEV entran en la categoría de consumidores vulnerables.

Para llevar a cabo la investigación se ha realizado una encuesta a los socios de Som Energia. Som Energia es la mayor CEV de España, dado que cuenta con más de 22.000 socios procedentes de todas las Comunidades Autónomas, gran parte de ellos en Cataluña. Para enviar la encuesta se ha utilizado la plataforma de Som Energia, ya que desde la cooperativa directamente no había la posibilidad de enviar el cuestionario a los socios. En la plataforma estaban inscritos 1.656 socios, a los que se les envía la encuesta en agosto de 2015 y una breve explicación sobre cómo realizarla y los propósitos del estudio.

Las preguntas del cuestionario se formulan en torno a los requisitos de acceso a las tarifas sociales, los cuales están definidos en legislación, el proyecto de ley y el convenio que dan lugar a cada una de esas tarifas. Para la elaboración del cuestionario se recurrió a la herramienta de formularios de Google. En primer lugar se envió un borrador del cuestionario a un número limitado de socios. Tras obtener las primeras respuestas, el cuestionario se modificó a fin de aclarar conceptos y facilitar las respuestas. En esta etapa también se contó con la colaboración del director de la CEV GoiEner.

El cuestionario final queda conformado por dos grandes bloques: uno para trazar el perfil socioeconómico del socio de la CEV, y otro para recabar información entorno al cumplimiento de los requisitos de acceso a las tarifas sociales (Anexo I).

\section{RESULTADOS Y DISCUSIÓN}

La tasa de respuesta al cuestionario es del 10,62\% de la muestra, habiendo respondido un total de 176 socios. La muestra es representativa para los usuarios de la plataforma para un error muestral del 7\% y un nivel de confianza del 95\%, pero no para el total de socios de la cooperativa. A continuación se describen y discuten los resultados para los dos objetivos planteados en el estudio.

\subsection{Perfil socioeconómico del socio}

Un 79\% de los encuestados fueron hombres y un 75\% tenía una edad comprendida entre los 30 y los 50 años. En la muestra están representadas 31 provincias, procediendo un $30 \%$ de los encuestados de la provincia de Barcelona. 
En un 34,2\% de los casos la unidad familiar consta de 2 miembros, mientras que en un $22,8 \%$ se compone de 4 miembros. Las familias de un solo miembro constituyen el $19 \%$ de la muestra, mientras que las de tres miembros alcanzan el 18,3\%. Por último, sólo en el 5,7\% de los casos la familia consta de 5 miembros o más.

El $77,7 \%$ de los encuestados cuentan con formación universitaria $(47,1 \%$ de diplomados, licenciados o ingenieros; $21,6 \%$ de posgrado o máster y $9 \%$ de doctores), un $11 \%$ de ellos tiene formación profesional, un $8,5 \%$ bachillerato y el resto, un $2,8 \%$, graduado escolar o ESO.

El perfil tipo del encuestado es el de un varón catalán de mediana edad que vive en pareja y que tiene estudios superiores. Los encuestados proceden principalmente de Barcelona, hecho que estaría explicado por el origen catalán de Som Energia. Respecto al tamaño de la unidad familiar, si bien predominan las familias de dos miembros, el porcentaje de familias de uno, tres y cuatro miembros es similar. Los resultados de la encuesta indirectamente ponen de manifiesto que la formación está estrechamente relacionada con la conciencia ambiental, ya que entre los socios de las CEV abundan aquellos que tienen una formación superior.

\subsection{Beneficiarios del bono o tarifa social en cada escenario}

En este apartado se mostraran los resultados obtenidos a partir de la segunda parte del cuestionario, de aspectos relacionados con el bono social, y se discutirá principalmente sobre los cuatro escenarios. Se verá qué porcentaje de encuestados accedería al bono social en cada escenario y sus características socioeconómicas.

\section{Escenario 1. Bono social actual}

Un $65 \%$ de los encuestados afirma tener conocimiento de la existencia del bono social. Tal y como se aprecia en el gráfico 1 , un $16 \%$ de los encuestados tendría derecho al bono social actual si tuvieran contratada la comercialización de la electricidad con una comercializadora de referencia. De este $16 \%$, el $71 \%$ sabe lo que es el bono social, pero prefiere renunciar a él y mantenerse en la cooperativa. El restante $29 \%$ no sabe lo que es el bono social. 
Gráfico 1. Derecho al bono social actual

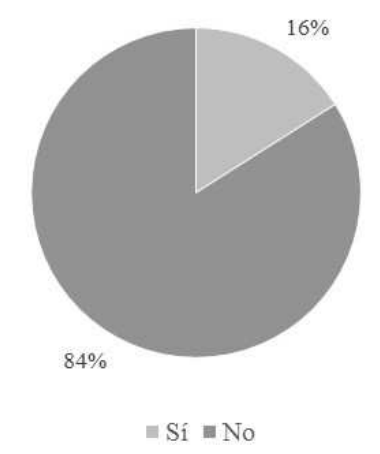

Fuente: elaboración propia

En este $16 \%$ que tendrían derecho al bono social, la mayoría de hogares accederían por tener contratada una potencia inferior a $3 \mathrm{~kW}(39 \%)$ o por ser familia numerosa (32\%), siendo estas causas seguidas por la condición de tener a todos sus miembros en paro (18\%). El gráfico 2 refleja estos datos.

Gráfico 2. Beneficiarios del bono social actual por requisito de acceso
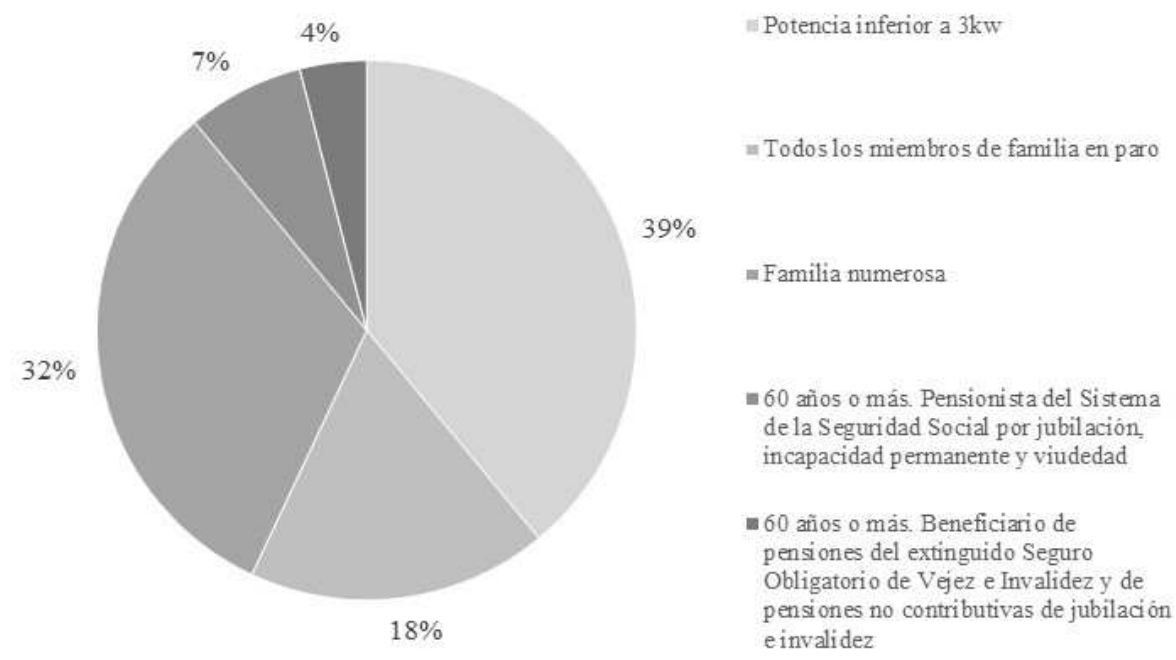

Fuente: elaboración propia

\section{Escenario 2. Proyecto de bono social con requisitos mixtos}

Un $6,25 \%$ de los encuestados accedería al bono social si este tuviera como requisitos de acceso las condiciones contempladas en el proyecto de bono social con requisitos mixtos (gráfico 3). 
Gráfico 3. Derecho al bono social con requisitos mixtos

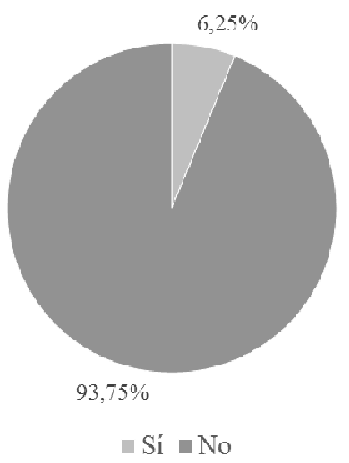

Fuente: elaboración propia

El gráfico 4 desagrega los beneficiarios según el requisito por el que acceden al bono social con requisitos mixtos. En este escenario casi la mitad de beneficiarios, el $45,45 \%$ de ellos, forman parte de unidades familiares con todos sus miembros en paro.

Gráfico 4. Beneficiarios del bono social con requisitos mixtos

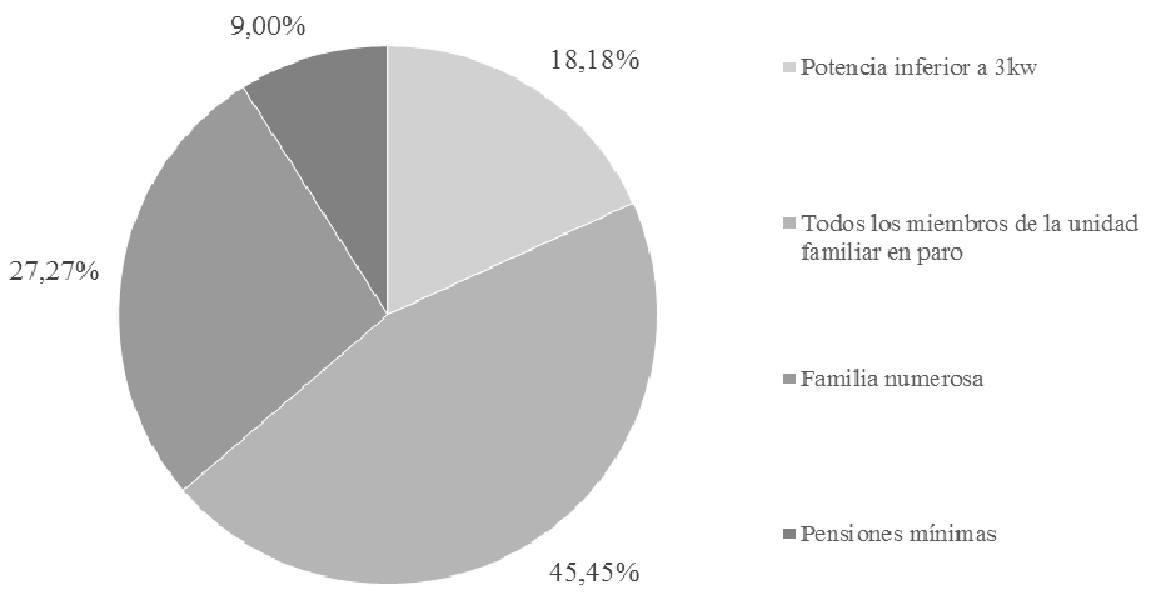

Fuente: elaboración propia

\section{Escenario 3. Bono social con requisitos económicos}

Si se tienen en cuenta los nuevos requisitos que se está estudiando aplicar al bono social, los cuales, además de tener en cuenta los requisitos actuales, incorporan el tamaño de la unidad familiar y sus ingresos brutos, el porcentaje de encuestados que tendría acceso al bono social con requisitos económicos incrementaría hasta el 20\% (gráfico 5). 
Gráfico 5. Derecho al bono social con requisitos económicos

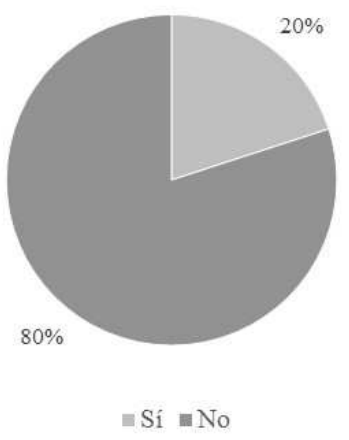

Fuente: elaboración propia

Tal y como se aprecia en el gráfico 6, en este escenario un $71 \%$ serían nuevos beneficiarios. Nuevo beneficiario es aquel que no cumple ninguno de los requisitos para acceder al bono social actual, pero que sí lo haría bajo las condiciones planteadas en este escenario. El 29\% de beneficiarios restantes estaría constituido por hogares que ya cualifican para el acceso al bono social actual: un $6 \%$ por tener contratada una potencia inferior a $3 \mathrm{~kW}$, el $11 \%$ por tener a todos los miembros de la familia en situación de desempleo, el $9 \%$ por ser familia numerosa y el 3\% por cobrar una pensión mínima.

Gráfico 6. Beneficiarios del bono social con requisitos económicos

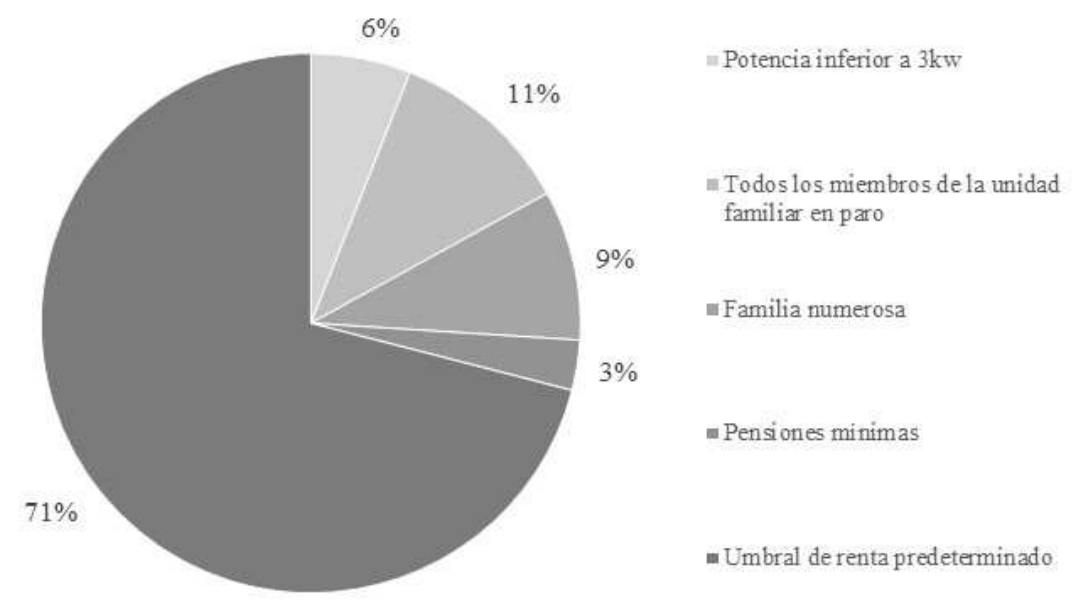

Fuente: elaboración propia

\section{Escenario 4. Tarifa social alternativa}

Para el cálculo de los requisitos de la tarifa social alternativa es necesario conocer los ingresos netos mensuales por unidad familiar una vez deducido el alquiler o la hipoteca hasta 
un máximo de $600 €$ mensuales. Para ello, se han convertido los ingresos brutos a ingresos netos mensuales (Anexo II). Este ingreso neto debe pasar los 227,64€/mes por miembro de la unidad familiar.

Un $12 \%$ de los encuestados accedería a esta tarifa social, lo que representa un $4 \%$ menos respecto al bono social actual (gráfico 7). Cabe destacar que de entre este $12 \%$ de socios potencialmente vulnerables, el $47 \%$ no sabe nada o solo ha oído algo del bono social.

Gráfico 7. Derecho a la tarifa social alternativa

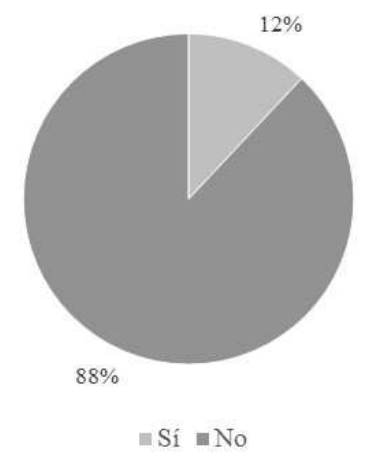

Fuente: elaboración propia

El gráfico 8 muestra que el $76 \%$ de los beneficiarios serían nuevos beneficiarios, mientras que un $14 \%$ de los beneficiarios tendría derecho al bono social actual al cumplir el requisito de que todos los miembros de la familia estén en el paro. El 10\% de los beneficiarios en el escenario de la tarifa social también accederían al bono social actual, pero por tener contratada una potencia inferior a $3 \mathrm{~kW}$. Ninguno de los que antes accedía al bono social por familia numerosa o por pensión mínima accede al bono social en este escenario. 
Gráfico 8. Beneficiarios de la tarifa social alternativa por requisito de acceso

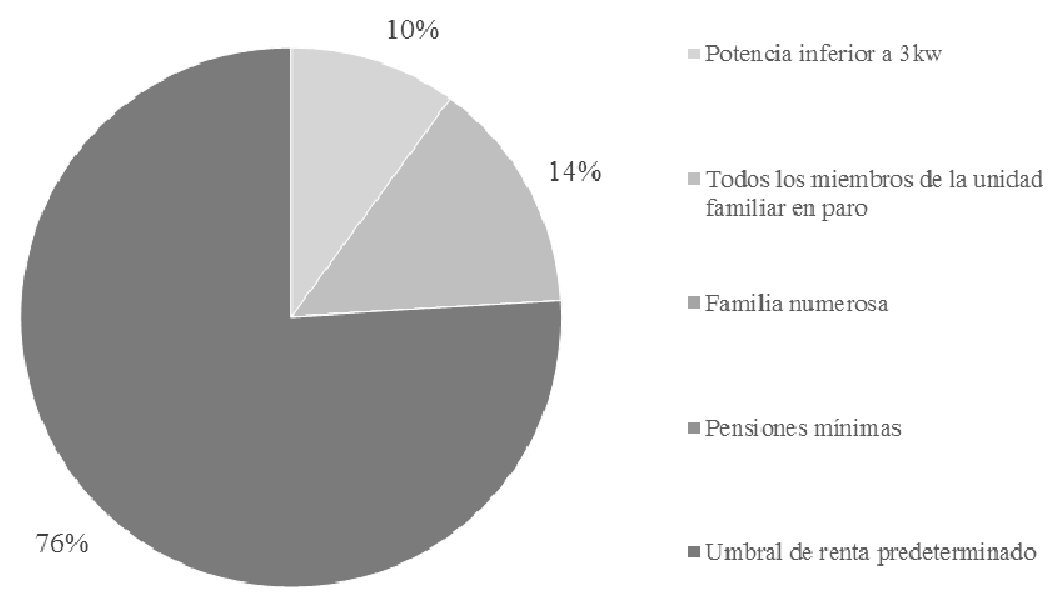

Fuente: elaboración propia

\section{Comparación de escenarios}

La tabla 5 compara el porcentaje de beneficiarios que tendrían derecho a acceder a bonificaciones en cada uno de los escenarios, así como el requisito por el que accederían.

Respecto al derecho a las bonificaciones, el mayor porcentaje de beneficiarios se da en el escenario de bono social con requisitos económicos (20\%), mientras que el menor porcentaje corresponde al escenario del bono social con requisitos mixtos $(6,25 \%)$. La mayoría de los beneficiarios en el escenario del bono social con requisitos económicos $(71 \%)$ $\mathrm{y}$ en el escenario de la tarifa social alternativa (76\%) no cumplen las condiciones socioeconómicas del bono social actual. 
Tabla 5. Comparación de escenarios

\begin{tabular}{|c|c|c|c|c|}
\hline Acceso & $\begin{array}{c}1 . \text { Bono social } \\
\text { actual }\end{array}$ & $\begin{array}{c}\text { 2. Proyecto de } \\
\text { bono social } \\
\text { con requisitos } \\
\text { mixtos }\end{array}$ & $\begin{array}{c}\text { 3. Bono social } \\
\text { con requisitos } \\
\text { económicos }\end{array}$ & $\begin{array}{c}\text { 3. Tarifa } \\
\text { social } \\
\text { alternativa }\end{array}$ \\
\hline \hline \multicolumn{5}{|c|}{ Derecho de acceso } \\
\hline Sí & $16 \%$ & $6,25 \%$ & $20 \%$ & $12 \%$ \\
\hline No & $84 \%$ & $93,75 \%$ & $80 \%$ & $88 \%$ \\
\hline \hline \multicolumn{5}{|c|}{ Requisito de acceso } \\
\hline $\begin{array}{c}\text { Potencia inferior a } \\
\text { 3kW }\end{array}$ & $39 \%$ & $18,18 \%$ & $6 \%$ & $10 \%$ \\
\hline Desempleados & $18 \%$ & $45,45 \%$ & $11 \%$ & $14 \%$ \\
\hline Familia numerosa & $32 \%$ & $27,27 \%$ & $9 \%$ & $0 \%$ \\
\hline Pensiones mínimas & $11 \%$ & $9 \%$ & $3 \%$ & $0 \%$ \\
\hline Umbral de renta & $0 \%$ & $100 \%$ & $71 \%$ & $76 \%$ \\
\hline
\end{tabular}

Fuente: elaboración propia

Los resultados muestran la diferencia entre el perfil del beneficiario del bono social actual y el que perfil del beneficiario que surgiría si se aplicaran criterios de acceso más ajustados a la realidad resultante tras la crisis. En cuanto al apartado de los requisitos de acceso, las cifras que presentan reflejan la participación relativa de cada grupo de beneficiarios en el total de hogares con derecho a la tarifa social, según cada escenario.

Respecto al escenario del bono social actual, la incorporación del requisito de umbral de renta en el escenario de bono social con requisitos mixtos reduciría el porcentaje de beneficiaros del $16 \%$ al 6,25\%. Los principales afectados por tal reducción serían los hogares que contratan una potencia inferior a $3 \mathrm{~kW}$, cuya contribución al total de beneficiarios bajaría del $39 \%$ al $18,18 \%$, y los beneficiarios por familia numerosa, que pasaría del $31 \%$ al $27,7 \%$. Por el contrario, aumentaría desde el $18 \%$ hasta el $45,45 \%$ el porcentaje de los beneficiaros cuya unidad familiar tiene a todos sus miembros en paro. Por tanto, la potencia contratada y la condición de familia numerosa deberían ser requisitos secundarios para acceder al bono social, priorizándose el umbral de renta.

Además, en todos los escenarios alternativos al del bono social actual se reduce drásticamente el número de beneficiarios que cumplen el requisito de acceso de potencia inferior a $3 \mathrm{~kW}$. Desde el escenario del bono social actual, en el que los beneficiarios que cumplirían este requisito serían la mayoría (39\%), se pasaría a porcentajes relativos muy inferiores: $18,18 \%$ en el escenario del proyecto de bono social con requisitos mixtos, $6 \%$ en el 
escenario del bono social con requisitos económicos y $10 \%$ en el escenario de tarifa social alternativa. Los escenarios alternativos evidencian que contratar una potencia baja no implica necesariamente que se esté en situación de vulnerabilidad económica.

Todas estas cifras ponen evidencian la vulnerabilidad, sobre todo en lo que respecta al requisito de umbral de renta, de los socios de CEV, además de poner de manifiesto la importancia de ajustar los requisitos de acceso al bono social para que dé cobertura a consumidores vulnerables desatendidos.

\section{CONCLUSIONES}

En los últimos años, el problema de la pobreza energética se ha agravado, especialmente debido a la pérdida de poder adquisitivo de las familias. Al mismo tiempo, ha irrumpido en el sector eléctrico el fenómeno de las CEV. Todo ello en un contexto en el que ni el bono social actual contempla requisitos económicos generalizados de acceso, ni las CEV tienen la obligación de proporcionar una tarifa social a sus socios. Este trabajo contribuye a conocer la incidencia de la pobreza energética en hogares que contratan su electricidad con las CEV.

En principio, dado el perfil socioeconómico del socio consumidor de las CEV, podría pensarse que la exposición a la pobreza energética de los socios de estas cooperativas es menor. Adicionalmente, en los resultados se observa que hay cooperativistas que, aun teniendo derecho al bono social actual y conociendo su existencia, renuncian a él en pro de formar parte del proyecto sostenible de las CEV.

Sin embargo, el cálculo de porcentaje de beneficiarios por escenario refleja que sí existe pobreza energética en las CEV. Este problema podría paliarse destinando una parte de los beneficios, en el caso de que los haya, a sufragar la factura de aquellos socios vulnerables. No obstante, hasta que las cooperativas adquieran un tamaño considerable, esta solución resulta difícil.

A pesar de la necesidad de concienciar sobre la importancia del uso de energía renovable en todos los segmentos de la población, la inexistencia de una tarifa social en las $\mathrm{CEV}$ supone un freno que afecta especialmente a las familias con ingresos bajos. Este obstáculo podría superarse fomentando políticas públicas que ayuden a las CEV a ofrecer una 
bonificación equivalente a los consumidores vulnerables que actualmente no pueden permitírsela.

En cualquier caso, redefinir el concepto de consumidor vulnerable parece una prioridad. Actualmente, las cooperativas tienen la ventaja de que pueden fijar sus criterios socioeconómicos para definirlo. La crisis ha tenido un gran efecto negativo en la capacidad adquisitiva de las familias, por lo que los requisitos que establece el Real Decreto-ley 6/2009, de 30 de abril para acceder al bono social han quedado desfasados. Por tanto, son necesarios cambios legislativos que introduzcan requisitos de renta para acceder al bono social, sin perjuicio de que puedan seguir aplicándose requisitos socioeconómicos adicionales.

Finalmente, no ha de olvidarse que las medidas de eficiencia energética también sirven para combatir el problema de la pobreza energética En este sentido, la educación en torno al buen consumo energético es un aspecto clave, ya que enseña al consumidor cómo ahorrar energía, por ejemplo, adecuando la potencia contratada a sus necesidades de consumo o valorando el ahorro potencial que le brindaría una tarifa de discriminación horaria.

\section{BIBLIOGRAFÍA}

ARNÁEZ ARCE, V.M. (2011) La comercialización de energía eléctrica a través de cooperativas de consumo. Boletín de la Asociación Internacional de Derecho Cooperativo, $\mathrm{n}^{\mathrm{o}} 45$, pp. 197-216.

CNMC (2014) ¿Cuántos usuarios se benefician del bono social de electricidad en España? Comisión Nacional de los Mercados y la Competencia.

CNMC (2015) Listado de comercializadoras de energía eléctrica. Comisión Nacional de los Mercados y la Competencia.

MARÍN URIBE, P. (2009) El déficit de tarifa. Cuadernos de energía, no 25, pp. 34-36.

MINET (2015). Listado público de distribuidoras eléctricas. Ministerio de Industria, Energía y Turismo.

MORENO, A., PÉREZ, D. Y URIS, S. (2014) Asuntos relevantes para la sostenibilidad del sector eléctrico. Fase II: Análisis de las políticas y la regulación. Septiembre 2013 Febrero 2014. Energía y sociedad - Grupo de investigación en Organizaciones Sostenibles. 
PÉREZ LUCÍA, J. (2015) La Cooperativa eléctrica de Museros. Disponible en: http://www.muserosfluidoelectrico.com/la-cooperativa-electrica-de-museros/ (último acceso: 03/11/2015). Fuente:Archivo histórico de la Cooperativa Eléctrica de Museros.

ROMERO, J.C., LINARES, P Y LÓPEZ OTERO, X. (2014) Pobreza Energética en España, análisis económico y propuestas de actuación. Vigo: Economics for Energy.

TIRADO HERRERO, S., JIMÉNEZ MENESES, L., LÓPEZ FERNÁNDEZ, J.L. Y MARTÍN GARCÍA, J. (2014) Pobreza energética en España: análisis de tendencias. Madrid: Asociación de Ciencias Ambientales.

\section{FUENTES DOCUMENTALES}

ESPAÑA. Proyecto de Real Decreto por el que se regula la actividad de comercialización y las condiciones de contratación y suministro de energía eléctrica de 19 de julio de 2013. Secretaría de Estado de Energía, Ministerio de Industria, Energía y Turismo.

ESPAÑA. Real Decreto-ley 6/2009, de 30 de abril, por el que se adoptan determinadas medidas en el sector energético y se aprueba el bono social. Boletín Oficial del Estado, núm. 111, de 7 de mayo de 2009, páginas 39404 a 39419.

ESPAÑA. Real Decreto 216/2014, de 28 de marzo, por el que se establece la metodología de cálculo de los precios voluntarios para el pequeño consumidor de energía eléctrica y su régimen jurídico de contratación. Boletín Oficial del Estado, núm. 77, de 29 de marzo de 2014, páginas 27397 a 27428.

GIRONA. Conveni Entre L'Ayuntament de Caldes de Montbui i L'Electra Caldense Energia, S.A. per al Desenvolupamente DÁccions de Suport Social a Consumidors D’Energia Elèctrica. Documento facilitado por la empresa Electra Caldense S.A.

UNIÓN EUROPEA. Dictamen de Iniciativa 2013/C 341/05 del Comité Económico y Social Europeo de 21 de noviembre de 2013 sobre el tema "Por una acción europea coordinada para prevenir y combatir la pobreza energética”. Diario Oficial de Unión Europea, 21 de noviembre de 2013.

UNIÓN EUROPEA. Directiva 2009/72/CE del Parlamento Europeo y del Consejo de 13 de julio de 2009 sobre normas comunes para el mercado interior de la electricidad y por la que se deroga la Directiva 2003/54/CE. Diario Oficial de Unión Europea, 13 de julio de 2009. 


\section{ANEXO I. Cuestionario de la encuesta}

\section{Bloque I. Perfil socioeconómico del socio}

I.1. Sexo

- Masculino

- Femenino

I.2. Edad

- Menos 30 años

- 41-45 años

- 56-60 años

- 30-35 años

- 46-50 años

- Más de 60 años

- 36-40 años

- 51-55 años

I.3. Provincia de residencia: 50 opciones (una por provincia)

I.4. Formación académica

- Graduado escolar o ESO

- Bachillerato

- Diplomatura, licenciatura o

- Formación profesional ingeniería

- Posgrado

- Doctorado

I.5. Tamaño de la unidad familiar.

- 1 miembro

- 3 miembros

- 5 miembros

- 2 miembros

- 4 miembros

- 6 miembros o más

\section{Bloque II. Requisitos de acceso a tarifas sociales}

II.1. ¿Sabe lo que es el bono social eléctrico?

- Sí

- He oído algo sobre ello pero no sé exactamente lo que es.

- No.

II.2. ¿Cumple alguno de estos requisitos de acceso al bono social?

- Tener 60 o más años de edad y ser pensionista del Sistema de la Seguridad Social por jubilación, incapacidad permanente y viudedad, percibiendo por ello la cuantía mínima vigente en cada momento para dichas clases de pensión con respecto a los titulares con cónyuge a cargo o a los titulares sin cónyuge que viven en una unidad económica unipersonal.

- Tener 60 o más años de edad y ser beneficiario de pensiones del extinguido Seguro Obligatorio de Vejez e Invalidez y de pensiones no contributivas de jubilación e invalidez. 
- Ser familia numerosa.

- Formar parte de una unidad familiar que tenga a todos sus miembros en situación de desempleo.

- Potencia contratada inferior a $3 \mathrm{~kW}$.

- No, ninguno

II.3. ¿Cuánta potencia tiene contratada?

$$
\begin{aligned}
& \text { - } 1,15 \mathrm{~kW} \\
& \text { - } 4,6 \mathrm{~kW} \\
& \text { - Más de 6,9kW } \\
& \text { - } 2,3 \mathrm{~kW} \\
& \text { - } 5,75 \mathrm{~kW} \\
& \text { - } 3,45 \mathrm{~kW} \\
& \text { - } 6,9 \mathrm{~kW}
\end{aligned}
$$

II.4. ¿A cuánto ascienden los ingresos brutos anuales de la unidad familiar?

- Menos de $10.841 €$

- Entre $10.841 €$ y $18.429 €$

- Entre $18.429 €$ y $23.849 €$

- Entre $23.849 €$ y $29.269 €$
- Entre $29.269 €$ y $34.689 €$

- Entre $34.689 €$ y $40.109 €$

- Más de 40.109€

II.5. ¿A cuánto asciende el coste anual de la hipoteca o del alquiler de la vivienda habitual?

- Menos de $3.600 €$

- Entre $3.600 €$ y $5.400 €$

- Entre $5.400 €$ y $7.200 €$

- Más de $7.200 €$

\section{ANEXO II. Correspondencia entre euros brutos anuales y euros netos mensuales}

Euros brutos anuales Menos de $10.841 €$

Entre $10.841 €$ y $18.429 €$ Entre $18.429 €$ y $23.849 €$ Entre $23.849 €$ y $29.269 €$ Entre $29.269 €$ y $34.689 €$ Entre $34.689 €$ y $40.109 €$ Más $40.109 €$
Euros netos mensuales* $\rightarrow \quad$ Menos de 782,81€

$\rightarrow \quad$ Entre $782,81 €$ y $1.223,22 €$

$\rightarrow \quad$ Entre $1.223,22 €$ y $1.523,35 €$

$\rightarrow \quad$ Entre $1.523,35 €$ y $1.820,78 €$

$\rightarrow \quad$ Entre $1.820,78 €$ y $2.100,13 €$

$\rightarrow \quad$ Entre $2.100,13 €$ y $2.361,42 €$

$\rightarrow \quad$ Más de 2.361,42 €

* Los cálculos se han realizado con la ayuda de un conversor de sueldo bruto a sueldo neto. Para simplificar el cálculo:

- No se ha tenido en cuenta el número de hijos. 
- La situación laboral utilizada para la conversión ha sido la de empleado indefinido. Teniendo en cuenta que existen familias con todos sus miembros en situación de desempleo, este supuesto es el más conservador.

- El tipo de contribuyente elegido es el tipo C.

A partir de aquí se ha tenido en cuenta la situación menos favorable: el ingreso mínimo declarado en cada tramo y el alquiler o el gasto hipotecario declarado más alto en cada tramo. Al ingreso mensual se le ha restado el gasto mensual del alquiler o de la hipoteca y ello se ha dividido por el número de personas que componen la unidad familiar. Por tanto, las cifras de beneficiarios están calculadas como el porcentaje máximo de encuestados que tendrían derecho a una tarifa social alternativa con las características ofrecidas por la empresa Electra Caldense Energia S.A. 